\title{
(-)-Epigallocatechin-3-gallate suppresses liver metastasis of human colorectal cancer
}

\author{
TAKEHITO MARUYAMA, SOICHIRO MURATA, KEN NAKAYAMA, NAOKI SANO, \\ KOICHI OGAWA, TAKESHI NOWATARI, TAKAFUMI TAMURA, REIJI NOZAKI, \\ KIYOSHI FUKUNAGA and NOBUHIRO OHKOHCHI
}

Department of Surgery, Graduate School of Comprehensive Human Sciences, University of Tsukuba, Tsukuba, Ibaraki 305-8575, Japan

Received September 26, 2013; Accepted November 11, 2013

DOI: $10.3892 /$ or.2013.2925

\begin{abstract}
Epigallocatechin-3-gallate (EGCG), the major constituent of green tea, has been shown to inhibit cell proliferation and induce apoptosis in several types of human tumors. The most common site of distant metastases in colorectal cancer is the liver. However, no previous studies have reported the ability of EGCG to suppress liver metastases of human colorectal cancer. The aim of the present study was to elucidate the potential use of EGCG as chemotherapy targeting liver metastases of human colorectal cancer. To assess the effect of EGCG on human colorectal cancer cell lines, RKO and HCT116, cell viability, cell proliferation and apoptosis were measured by Cell Counting kit-8, BrdU assay and TUNEL staining, respectively. Protein and gene expression were measured by western blot analysis and RT-PCR analysis, respectively. EGCG inhibited cell proliferation and induced apoptosis. EGCG dephosphorylated constitutively activated Akt and increased the activation of p38. EGCG also decreased the expression of vascular endothelial growth factor receptor 2. Additionally, the ability of EGCG to prevent the development of liver metastases of RKO tumors was evaluated in SCID mice. EGCG suppressed angiogenesis and induced apoptosis in liver metastases without associated body weight loss or hepatotoxicity. Furthermore, the liver metastatic area was significantly reduced by EGCG administration. Our findings indicate that EGCG may be useful in the treatment of liver metastases of human colorectal cancer.
\end{abstract}

\section{Introduction}

Colorectal cancer is one of the most common types of cancer and is responsible for $10 \%$ of all cancer-related mortality

Correspondence to: Professor Nobuhiro Ohkohchi, Department of Surgery, Graduate School of Comprehensive Human Sciences, University of Tsukuba, 1-1-1 Tennodai, Tsukuba, Ibaraki 305-8575, Japan

E-mail: nokochi3@md.tsukuba.ac.jp

Key words: (-)-epigallocatechin-3-gallate, liver metastasis, colorectal cancer, apoptosis, Akt, p38, angiogenesis worldwide (1). The liver is the most common site of distant metastasis in colorectal cancer. Approximately $15 \%$ of patients have liver metastases when the primary tumor is diagnosed (2), and half of patients ultimately develop liver metastases during the course of colorectal cancer. Colorectal cancer is unique among solid tumors in that the surgical resection of distant metastases, such as those in the liver and lung, can offer long-term survival in selected patients (3). Indeed, hepatic resection is the only curative treatment and is the standard therapy for liver metastases of colorectal cancer. However, only $25 \%$ of patients with liver metastases are candidates for liver resection based on tumor number, size and location (4), and one-third of patients treated with curative liver resection experience a recurrence in the liver $(5,6)$. Systemic chemotherapy is the treatment of choice for patients with unresectable liver metastases (7). Although long-term survival is rare among patients treated only with chemotherapy (8), recent advances in systemic chemotherapy have prolonged the survival of patients with unresectable metastases (9). The development of new agents and the shift from monotherapy to combination chemotherapy have been key in improving the clinical outcome for patients with unresectable metastases.

Green tea, a common beverage worldwide, has been studied extensively for its health benefits, including its anticancer effects (10). Epidemiological studies have shown that green tea has a potential preventive effect against colorectal cancer $(11,12)$. The antiproliferative effect of green tea has been attributed to the biological activities of its polyphenol components. The principal polyphenols in green tea include (-)-epicatechin (EC), (-)-epicatechin-3-gallate (ECG), (-)-epigallocatechin (EGC) and (-)-epigallocatechin-3-gallate (EGCG). EGCG is the most abundant and accounts for half of tea polyphenols (13); it is also the major biologically active component that inhibits cell proliferation and induces apoptosis in colorectal cancer cells (14). Recent studies have revealed that EGCG exerts its antiproliferative effect, at least in part, by modulating the activities of various receptor tyrosine kinases and their multiple downstream signaling pathways, including the Akt signaling pathway, which controls the expression of the multiple target genes involved in cell proliferation and apoptosis (15). Additionally, EGCG activates stress signals, such as c-Jun $\mathrm{N}$-terminal kinase (JNK) and p38 mitogen-activated 
protein kinase (MAPK), and induces apoptosis in colorectal cancer cell lines (16). EGCG has also been reported to inhibit the growth of human colorectal cancer cells in subcutaneous xenograft models (17-19). However, no previous studies have reported the ability of EGCG to suppress liver metastases of human colorectal cancer. The aim of the present study was to extend the investigation of the potential use of EGCG to treat liver metastases of human colorectal cancer. We examined the effect of EGCG on cell proliferation and apoptosis in the human colorectal cancer cell lines, RKO and HCT116. Subsequently, we investigated the effect of EGCG on liver metastases of RKO tumors in vivo.

\section{Materials and methods}

EGCG. EGCG was obtained from Sigma (St. Louis, MO, USA). For use in cell culture, EGCG was dissolved in sterile water at a concentration of $1 \mathrm{mg} / \mathrm{ml}$ and stored at $-20^{\circ} \mathrm{C}$. For use in intraperitoneal (i.p.) injections, EGCG was dissolved in saline at a concentration of $6 \mathrm{mg} / \mathrm{ml}$ and stored at $4^{\circ} \mathrm{C}$.

Cell culture. The human colorectal cancer cell lines, RKO and HCT116, were tested for mycoplasma prior to use. All cancer cells were aliquoted and frozen in liquid nitrogen immediately upon receipt and used for the present experiments within 6 months of thawing. All cancer cells were cultured in Dulbecco's modified Eagle's medium (DMEM) (Wako Pure Chemical Industries, Ltd., Osaka, Japan) supplemented with $10 \%$ fetal bovine serum (FBS) (HyClone, Logan, UT, USA) and $1 \%$ penicillin and streptomycin (Invitrogen, Grand Island, NY, USA). All cultures were maintained at $37^{\circ} \mathrm{C}$ in a humidified atmosphere containing $5 \% \mathrm{CO}_{2}$.

Cell viability assay. To measure the cytotoxicity of EGCG against these cancer cells, a total of $3 \times 10^{3}$ cells in $100 \mu \mathrm{l}$ of DMEM supplemented with 10\% FBS were seeded into each well of a 96-well plate. Following overnight culture, EGCG was added at the specified concentrations. After $24 \mathrm{~h}$ of incubation, cell viability was measured by the level of mitochondrial activity in reducing 2-(2-methoxy-4-nitrophenyl)-3-(4-nitrophenyl)-5- (2,4-disulfophenyl)-2H-tetrazolium monosodium salt (WST-8) to formazan using a Cell Counting kit-8 (CCK-8) (Dojindo Laboratories, Kumamoto, Japan). The cells were incubated with the reagent as specified by the manufacturer's instructions. The plates were read at $\mathrm{A}_{450}$ on a spectrometer.

Cell proliferation assay. To measure the effect of EGCG on the proliferative activity of these cancer cells, a total of $3 \times 10^{3}$ cells in $100 \mu \mathrm{l}$ of DMEM supplemented with $10 \%$ FBS were seeded in each well of a 96-well plate. Following overnight culture, EGCG was added at the specified concentrations. After $24 \mathrm{~h}$ of incubation, cell proliferation was measured with a Cell Proliferation ELISA, BrdU (colorimetric) (Roche Diagnostics, Penzberg, Germany). The cells were incubated with the reagent as specified by the manufacturer and the plates were read at $\mathrm{A}_{350}$ on a spectrometer.

Apoptosis assay. To detect apoptosis in these cancer cells, a total of $6 \times 10^{4}$ cells in $1 \mathrm{ml}$ of DMEM supplemented with $10 \%$ FBS were seeded in each well of a Lab-Tek II Chamber Slide
(Nalge Nunc International, Tokyo, Japan). Following overnight culture, EGCG was added at the specified concentrations, and the cells were incubated for a further $4 \mathrm{~h}$. Apoptosis in treated cells and paraffin-embedded liver samples was evaluated with a DeadEnd ${ }^{\mathrm{TM}}$ Colorimetric TUNEL System (Promega, Madison, WI, USA) according to the manufacturer's recommendations.

Western blot analysis. For western blot analysis of these cancer cells, a total of $3 \times 10^{5}$ cells in $5 \mathrm{ml}$ of DMEM supplemented with $10 \%$ FBS were seeded into each well of a 6 -cm dish. After $48 \mathrm{~h}$ of culture, EGCG was added at the specified concentrations, and the cells were harvested $12 \mathrm{~h}$ later. Cell lysates were subjected to $10 \%$ sodium dodecyl sulfate-polyacrylamide gel electrophoresis and transferred to a nitrocellulose membrane (Millipore, Bedford, MA, USA). The following antibodies were used as primary antibodies: Akt (9272), phospho-Akt (9271), p38 (9212) and phospho-p38 (9211) (Cell Signaling Technology, Beverly, MA, USA). $\beta$-actin (4970) (Cell Signaling Technology) was used as the endogenous control. An anti-rabbit IgG horseradish peroxidase-conjugated antibody (7074) (Cell Signaling Technology) was used as the secondary antibody. Immunoblots were analyzed by enhanced chemiluminescence.

Total RNA extraction. For reverse transcription-polymerase chain reaction (RT-PCR), a total of $3 \times 10^{5}$ cells in $5 \mathrm{ml}$ of DMEM supplemented with 10\% FBS were seeded into each well of a $6-\mathrm{cm}$ dish and cultured for $48 \mathrm{~h}$. EGCG was added at the specified concentrations, and after $2 \mathrm{~h}$ of incubation, total RNA was isolated from whole cells using a NucleoSpin ${ }^{\circledR}$ RNA II kit (Macherey-Nagel, Düren, Germany) according to the manufacturer's instructions. RNA concentrations were determined by measuring the absorbance at $260 / 280 \mathrm{~nm}$ with a NanoDrop Spectrophotometer (NanoDrop Technologies, Wilmington, DE, USA). The synthesis of complementary DNA was performed using AMV reverse transcriptase (Promega) and random primers (Takara Bio Inc., Shiga, Japan). Briefly, a mixture of $1 \mathrm{mM}$ dNTPs (Fermentas Life Sciences, Burlingame, ON, Canada), $0.025 \mu \mathrm{g} / \mathrm{ml}$ random primers, $0.25 \mathrm{U} / \mathrm{ml}$ reverse transcriptase, and $500 \mathrm{ng}$ of total RNA was incubated at $30^{\circ} \mathrm{C}$ for $10 \mathrm{~min}, 37^{\circ} \mathrm{C}$ for $60 \mathrm{~min}, 95^{\circ} \mathrm{C}$ for $5 \mathrm{~min}$ and at $4^{\circ} \mathrm{C}$ before storage at $-80^{\circ} \mathrm{C}$.

Quantitative real-time RT-PCR. Primers for RT-PCR were designed using Primer Express ${ }^{\circledR}$ software for Real-Time PCR ver. 3.0 (Applied Biosystems, Foster City, CA, USA) based on sequences available in GenBank. Primers were purchased from Hokkaido System Science (Hokkaido, Japan). We examined 4 receptor tyrosine kinases: vascular endothelial growth factor receptor 2 (VEGFR2), epidermal growth factor receptor (EGFR), human EGFR-related 2 (HER2) and c-Met. Glyceraldehyde-3-phosphate dehydrogenase (GAPDH) was used as an endogenous control. RT-PCR was performed using SYBR-Green real-time PCR Master Mix-Plus (Toyobo, Osaka, Japan) and an Applied Biosystems 7300 real-time PCR system (Applied Biosystems) as recommended by the manufacturers. The following primers were used for amplification: VEGFR2, 5'-TTGCCCTTGTTCTGTCCTTTTT-3' and 5'-GTCATTGT TCCCAGCATTTCAC-3'; EGFR, 5'-GGCTGCCTCCTGGA 
A

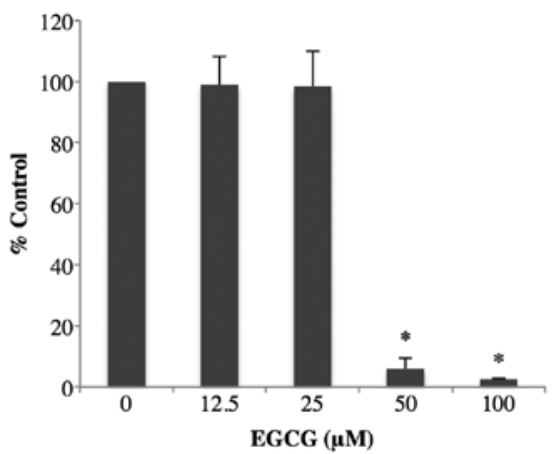

C

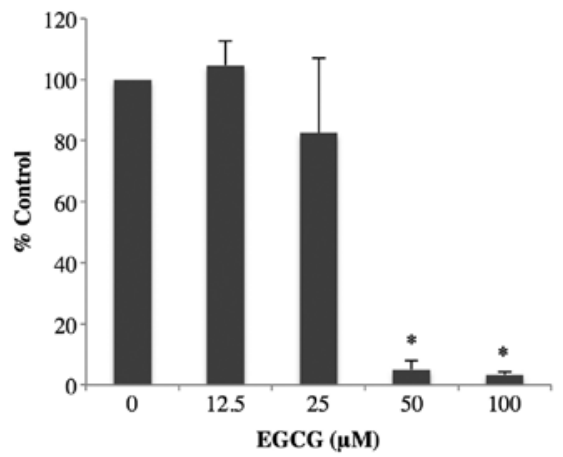

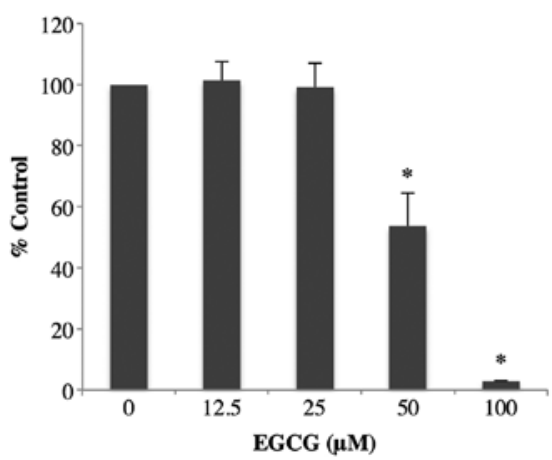

D

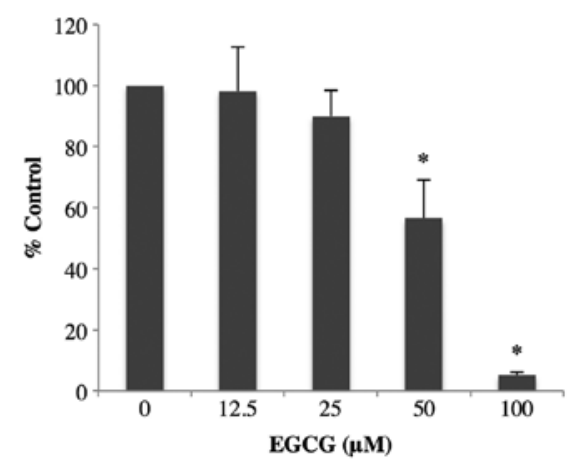

Figure 1. EGCG inhibits cell proliferation in colorectal cancer cells. Colorectal cancer cells were treated with 0-100 $\mu$ M EGCG. (A) CCK-8 of RKO; (B) CCK-8 of HCT116; (C) BrdU of RKO; and (D) BrdU of HCT116. Data are expressed as the means \pm standard deviation with $\mathrm{n}=8$ for each group. The values indicate the ratio compared to $0 \mu \mathrm{M}$ EGCG as $100 \%$. ${ }^{*} \mathrm{P}<0.01$ compared to $0 \mu \mathrm{M}$ EGCG.

CTATGT-3' and 5'-AGTTCATGCCCTTTGCATCT-3'; HER2, 5'-TCCCCCAAAGCCAACAAAG-3' and 5'-CCGTGGATGT CAGGCAGAT-3'; c-Met, 5'-CTCTCTGCCCCACCCTT TG-3' and 5'-TGTCCCGCTCAGGCATTC-3'; and GAPDH, 5'-GGAGTCCACTGGCGTCTTCA-3' and 5'-TTCACACCC ATGACGAACATG-3'.

Animal model of liver metastasis. All animal experiments were conducted in a humane manner in accordance with the Regulation for Animal Experiments of the University and Fundamental Guidelines for Proper Conduct of Animal Experiments and Related Activities in Academic Research Institutions under the jurisdiction of the Ministry of Education, Culture, Sports, Science and Technology of Japan, and were approved by the Institutional Animal Experiment Committee of the University of Tsukuba. All surgery was performed under isoflurane anesthesia and all efforts were made to minimize suffering.

Eight-week-old male severe combined immunodeficiency (SCID) mice weighing 21-26 g (CLEA Japan, Tokyo, Japan) were utilized in all experiments. The mice were housed in a temperature-controlled room with a 12-h light-dark cycle. They had free access to water and standard chow throughout the experiment. After an acclimation period of at least 7 days, laparotomy was performed with midline incision, and hepatic ischemia was induced by clamping the portal triad (hepatic artery, portal vein and bile duct) with a microclip (Aesculap, Tuttlingen, Germany) for $1 \mathrm{~min}$. At $1 \mathrm{~min}$ after reperfusion of the liver, $2 \times 10^{6}$ RKO cells were injected into the lower splenic pole with a 27 -gauge needle, and splenectomy was performed to prevent peritoneal dissemination from the spleen tumor. The mice were separated into two groups; the control group remained untreated $(n=12)$, and the EGCG group was treated with EGCG $(n=12)$. In the EGCG group, we administered EGCG (30 mg/kg body weight) by i.p. injection every other day over a 2-week period beginning 1 week after inoculation. At 21 days after inoculation, the mice were sacrificed and their livers were removed for examination.

Histological examination. To assess the liver metastatic area, all lobes of the liver were divided into 2 pieces, fixed with $10 \%$ neutral buffered formaldehyde. The tissues were embedded in paraffin and cut into $4 \mu \mathrm{m}$ sections. They were stained with hematoxylin-eosin, and the percentage of liver metastatic area was calculated using the image-processing software WinROOF (Mitani Co., Fukui, Japan).

Immunohistological examination. The paraffin-embedded tissues were deparaffinized with xylene, rehydrated with ethanol, immersed in $0.03 \%$ hydrogen peroxidase to block endogenous peroxidase activity, and then blocked with $3 \%$ bovine serum albumin (BSA) in phosphate-buffered saline to reduce background staining. An anti-CD31 antibody (LifeSpan Biosciences, Seattle, WA, USA) diluted 1:100 in 3\% BSA was added to the sections and incubated for $1 \mathrm{~h}$ at room temperature. They were then incubated with a peroxidase-labeled polymer conjugated to goat anti-rabbit immunoglobulins (Dako, Tokyo, Japan) for $30 \mathrm{~min}$ at room temperature, followed by diaminobenzidine chromogen (Dako) for $5 \mathrm{~min}$. The sections were counterstained with hematoxylin, dehydrated with ethanol, 
A

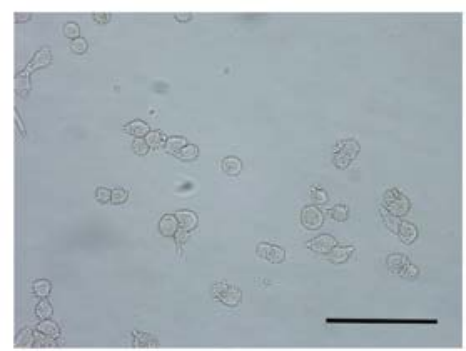

B

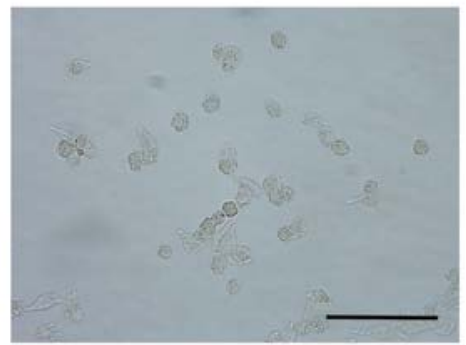

C

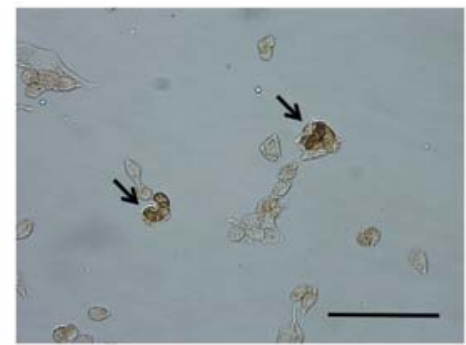

D

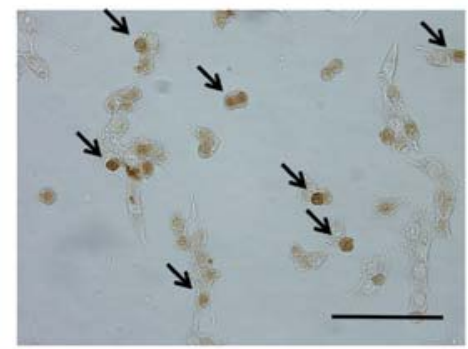

E

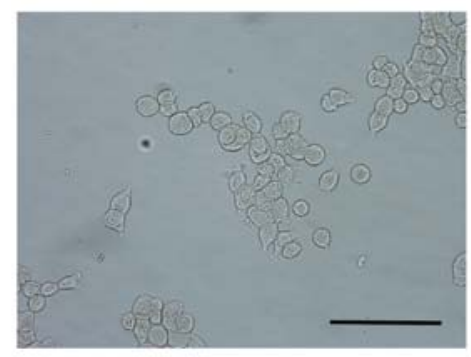

$\mathbf{F}$

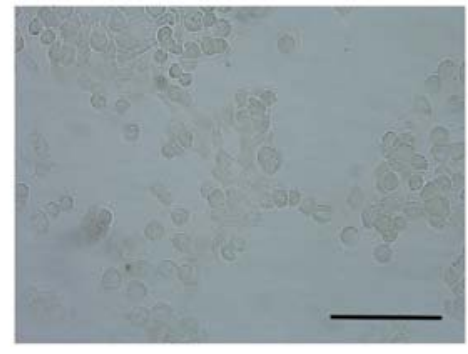

G

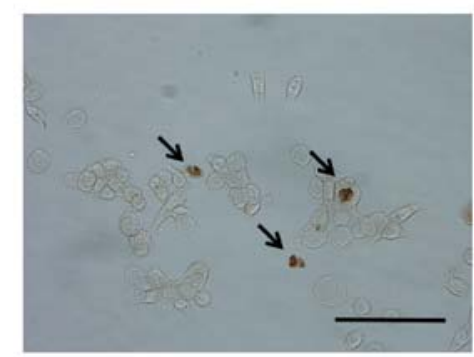

$\mathbf{H}$

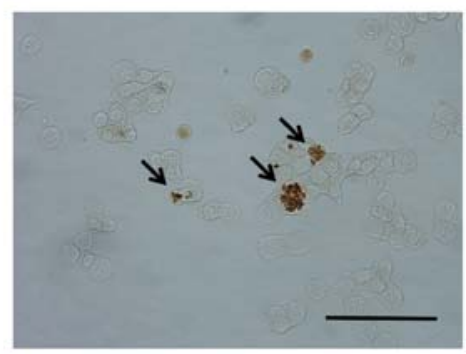

Figure 2. EGCG induces apoptosis in colorectal cancer cells. TUNEL staining of RKO cells treated with (A) EGCG $0 \mu \mathrm{M}$, (B) $25 \mu \mathrm{M}$, (C) $50 \mu \mathrm{M}$ and (D) $100 \mu \mathrm{M}$. The staining of HCT116 cells treated with (E) EGCG $0 \mu \mathrm{M}$, (F) $25 \mu \mathrm{M},(\mathrm{G}) 50 \mu \mathrm{M}$ and (H) $100 \mu \mathrm{M}$. Arrows indicate apoptotic colorectal cancer cells. Scale bars indicate $100 \mu \mathrm{m}$. Original magnification, $\mathrm{x} 400$.

made transparent with xylene and mounted with Multi Mount 480 (Matsunami Glass, Osaka, Japan).

Serum parameters. Blood was collected from the retro-orbital sinuses of the mice before sacrifice. The blood was centrifuged at $1,200 \mathrm{x} \mathrm{g}$ for $10 \mathrm{~min}$ at $4^{\circ} \mathrm{C}$. Supernatants were collected and stored at $-20^{\circ} \mathrm{C}$ until testing using a FUJI DRI-CHEM serum multiple biochemical analyzer (Fujifilm, Tokyo, Japan) to measure liver function, including aspartate aminotransferase (AST) and alanine aminotransferase (ALT).

Statistical analysis. All data are expressed as the means \pm standard deviation of samples. Unpaired t-test was used to compare two groups. Comparisons among more than three groups were performed using one-way analysis of variance with the Bonferroni post-test. In all cases, $\mathrm{P}<0.05$ was considered to indicate a statistically significant difference.

\section{Results}

Inhibition of colorectal cancer cell proliferation by EGCG. We initially determined whether EGCG treatment led to the inhibition of colorectal cancer cell proliferation. Colorectal cancer cells were treated with various doses of EGCG for $24 \mathrm{~h}$. Cell viability was assayed using the CCK-8 assay (Fig. 1A and B), and DNA synthesis was assessed using the BrdU assay (Fig. 1C and D). As shown in Fig. 1, EGCG at 50 and $100 \mu \mathrm{M}$ inhibited the proliferation of both colorectal cancer cells significantly compared to no EGCG treatment $(\mathrm{P}<0.01)$.

Induction of apoptosis by EGCG. The cancer cells were treated with various doses of EGCG for $4 \mathrm{~h}$, and cell apoptosis was detected by TUNEL staining. As shown in Fig. 2, TUNEL staining revealed that EGCG induced apoptosis in both colorectal cancer cells at 50 and $100 \mu \mathrm{M}$. 

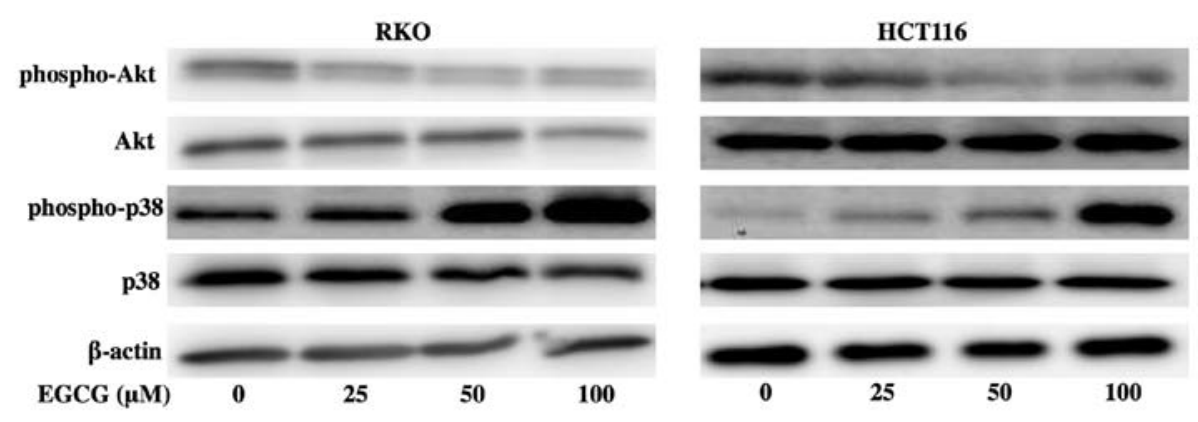

Figure 3. EGCG induces the dephosphorylation of Akt and the phosphorylation of p38. Colorectal cancer cells were treated with $0,25,50$ and $100 \mu \mathrm{M}$ of EGCG. After cell lysis, equal amounts of proteins were separated by SDS-PAGE, transferred to an Immobilon membrane and immunoblotted with antibodies against phospho-Akt, Akt, phospho-p38, p38 and $\beta$-actin. Left, RKO; right, HCT116.

A

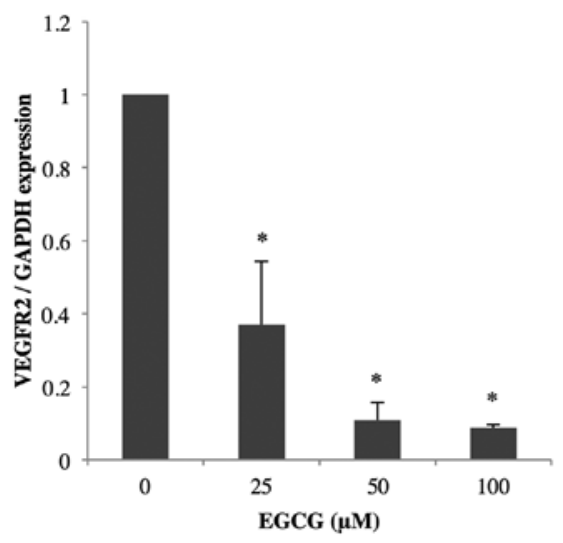

C

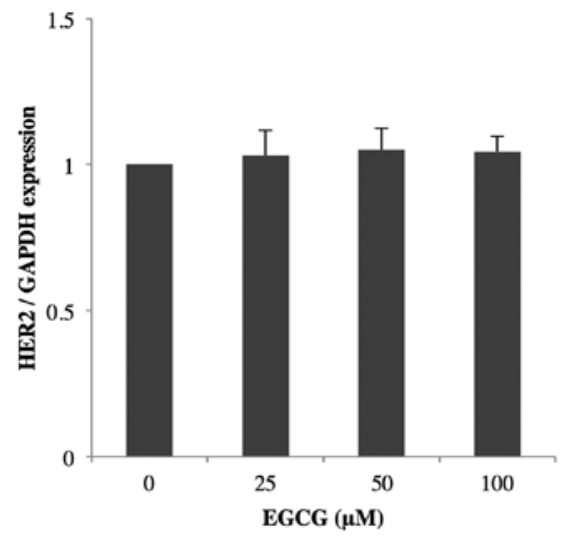

B

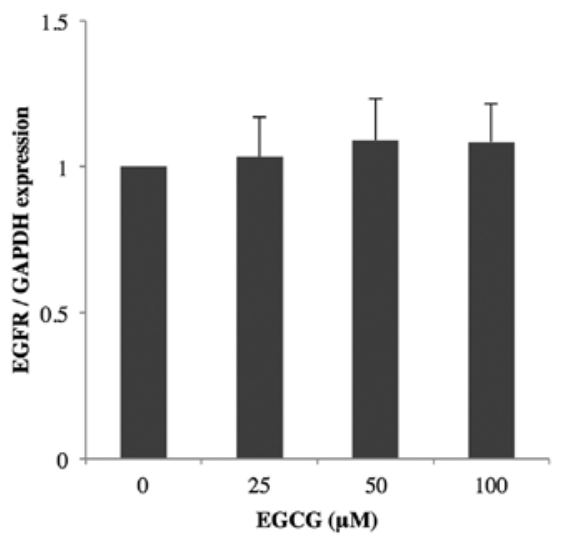

D

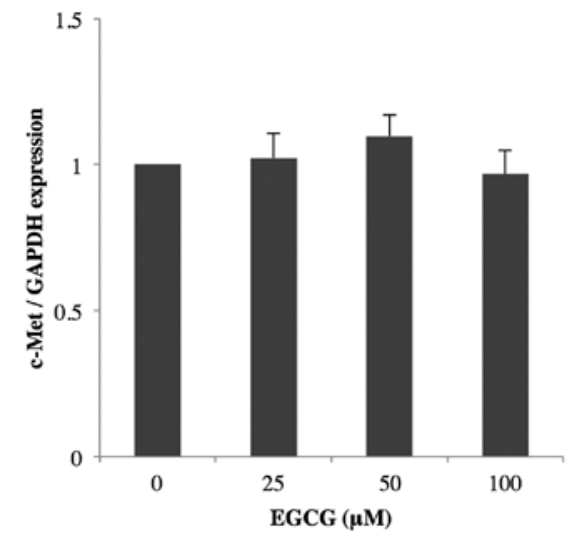

Figure 4. EGCG decreases the expression of VEGFR2 mRNA. RKO cells were treated with 0, 25, 50 and $100 \mu$ M EGCG. Total RNA was isolated from these cells, and the expression of (A) VEGFR2, (B) EGFR, (C) HER2 and (D) c-Met mRNAs was examined by quantitative real-time RT-PCR. Data are expressed as the means \pm standard deviation with $\mathrm{n}=4$ for each group. ${ }^{*} \mathrm{P}<0.01$ compared to $0 \mu \mathrm{M}$ EGCG.

The dephosphorylation of Akt and the phosphorylation of p38 by EGCG. In subsequent experiments, we examined the effects of EGCG on the expression of the signal transduction pathways in colorectal cancer cells. When RKO and HCT116 cells were treated with 25,50 or $100 \mu \mathrm{M}$ EGCG, there was the dephosphorylation of constitutive phosphorylation of Akt. In RKO cells, there was a marked increase in phospho-p38 at 50 and $100 \mu \mathrm{M}$ EGCG. In HCT116 cells, the activation of p38 occurred at $100 \mu \mathrm{M}$ EGCG (Fig. 3).

Decrease of VEGFR2 $m R N A$ by EGCG. We examined the effects of EGCG on the expression of VEGFR2, EGFR, HER2 and c-Met mRNAs in RKO cells. Quantitative real-time
RT-PCR indicated that treatment with 25,50 or $100 \mu \mathrm{M} \mathrm{EGCG}$ reduced the level of VEGFR2 mRNA significantly compared to no treatment; EGFR, HER2 and c-Met mRNAs were not affected by EGCG treatment (Fig. 4).

Suppression of liver metastases of human colorectal cancer by EGCG. To evaluate the effect of EGCG on liver metastases, we administered EGCG (30 mg/kg body weight) by i.p. injection every other day over a 2-week period beginning 1 week after RKO cell inoculation. Fig. 5A and B shows macroscopic findings and histological cross-sections of the livers removed from representative control and EGCG mice. A reduction in the growth of liver metastases was observed in the EGCG 
A

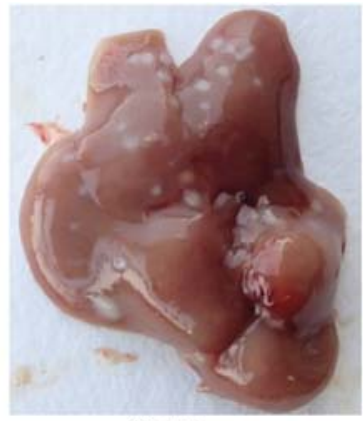

Control

B

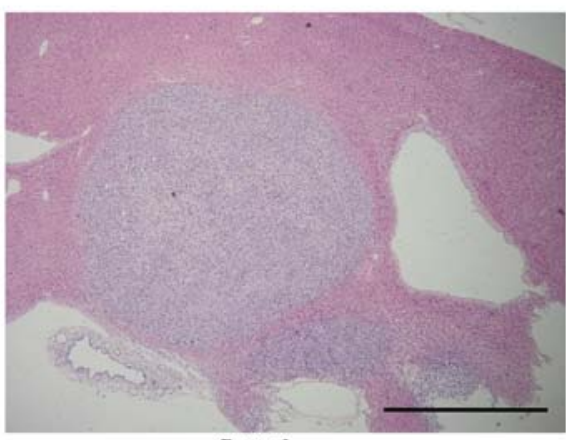

Control

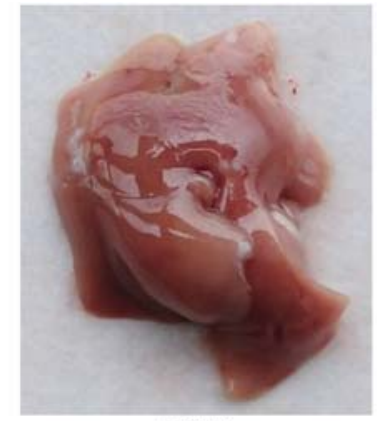

EGCG

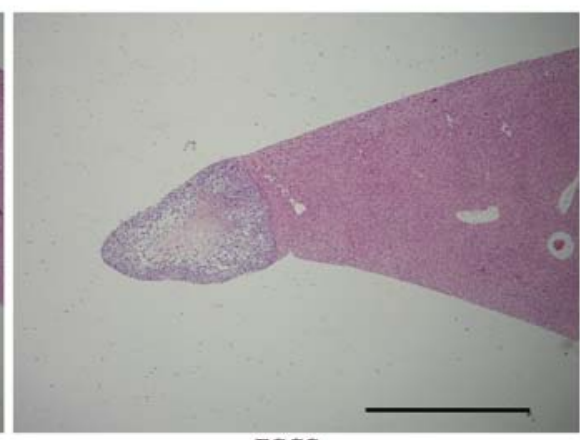

EGCG

C

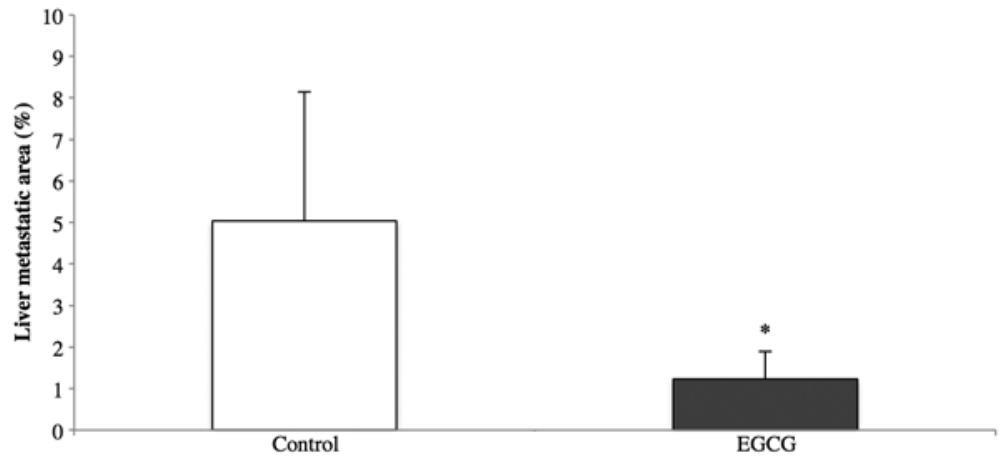

D

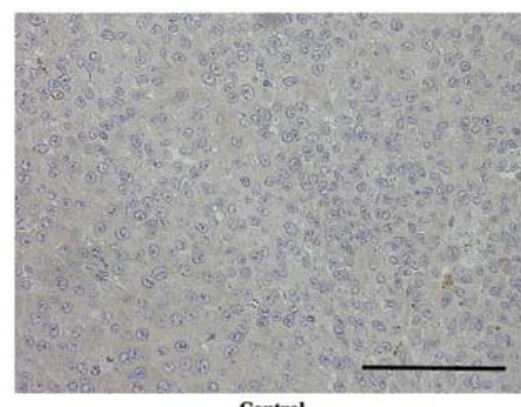

Control

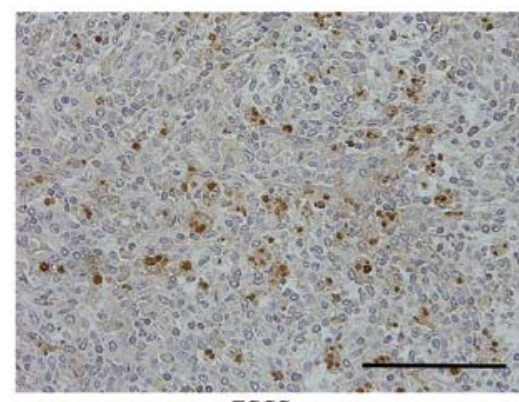

EGCG

$\mathbf{E}$
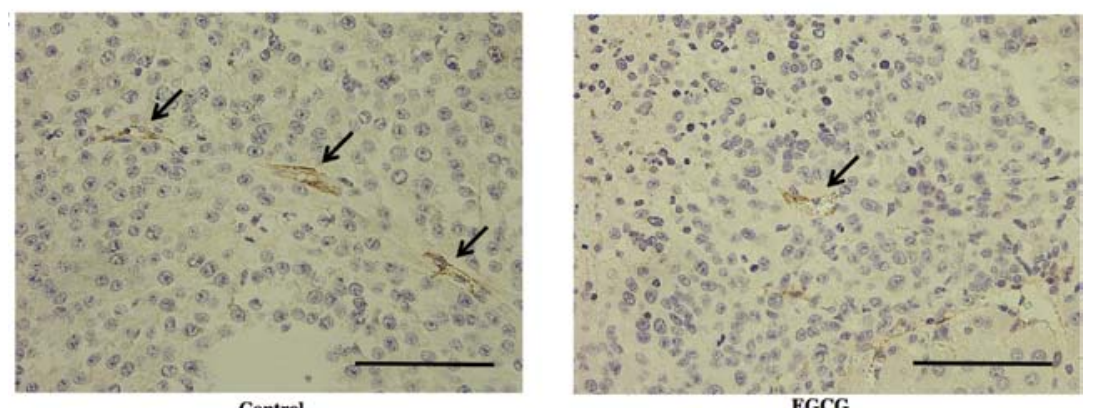

EGCG

Figure 5. EGCG suppresses liver metastases of human colorectal cancer. (A) Representative livers from the control and EGCG groups. (B) Histological cross-sections of livers from the control and EGCG groups. Scale bars indicate $1 \mathrm{~mm}$. Original magnification, $\mathrm{x} 40$. (C) Liver metastatic area in the livers of all mice in the control and EGCG groups. Data are expressed as the means \pm standard deviation with $n=12$ for each group. ${ }^{*}<0.05$ compared to the control group. (D) TUNEL staining of tumor tissues. Many TUNEL-positive apoptotic cells were observed in the EGCG group compared to the control group. Scale bars indicate $100 \mu \mathrm{m}$. Original magnification, x400. (E) Angiogenesis of liver metastases. Fewer tumor blood vessels were observed in the EGCG group than in the control group. Arrows indicate blood vessels stained with an anti-CD31 antibody. Scale bars indicate $100 \mu \mathrm{m}$. Original magnification, $\mathrm{x} 400$. 
A

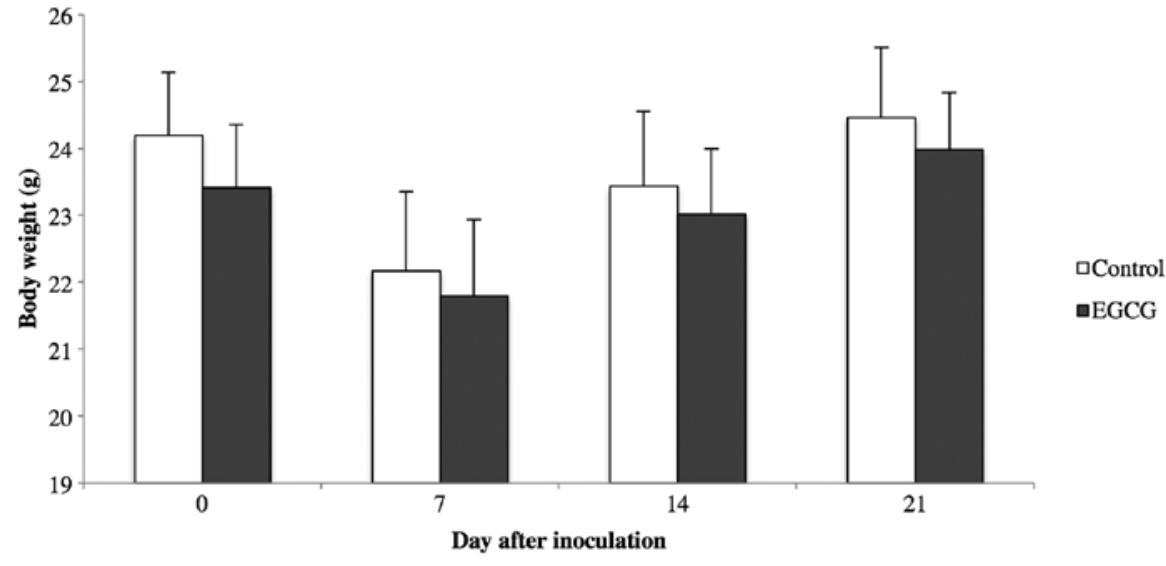

B

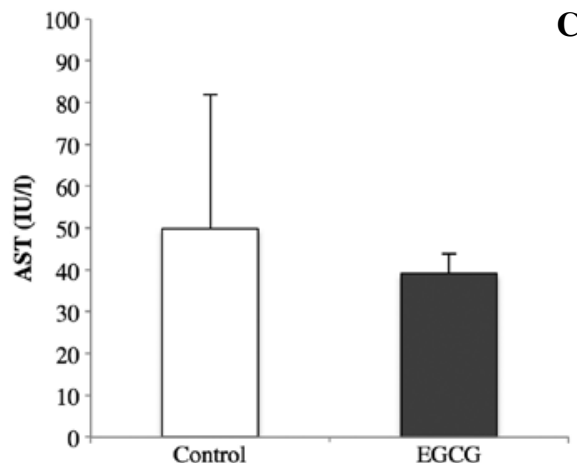

C

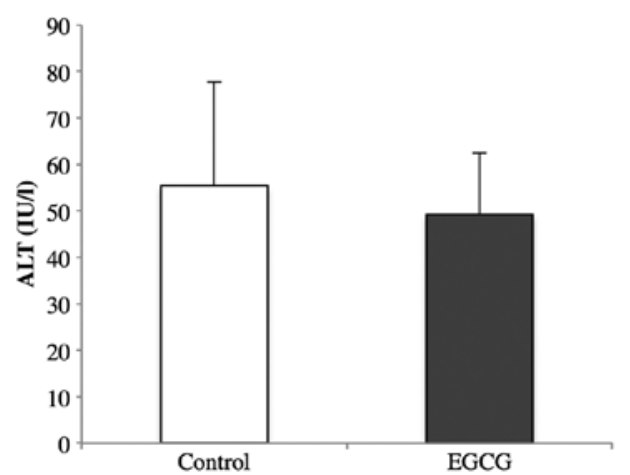

Figure 6. EGCG has no side-effects, such as weight loss or hepatotoxicity. (A) Body weight, (B) serum aspartate aminotransferase (AST) and (C) alanine aminotransferase (ALT) levels in the control and EGCG groups. Data are expressed as the means \pm standard deviation with $\mathrm{n}=12$ for each group.

group. Fig. 5C shows the tumor areas throughout the liver in both groups. Tumor growth was significantly reduced by EGCG administration. Fig. 5D shows the TUNEL staining of liver sections in the control and EGCG groups. In the EGCG group, apoptotic tumor cells were observed within the liver metastases. To further investigate whether EGCG inhibited angiogenesis, we used an anti-CD31 antibody to stain liver metastases. As shown in Fig. 5E, fewer tumor blood vessels were observed in the EGCG group than in the control group. No signs of toxicity, such as weight loss or elevated AST and ALT, were observed in mice treated with EGCG compared to the control mice (Fig. 6).

\section{Discussion}

The most common site of distant metastases in colorectal cancer is the liver. We extended the investigation of EGCG for the treatment of liver metastases of human colorectal cancer. In the present study, we revealed that EGCG inhibits cell proliferation and induces apoptosis in human colorectal cancer cells in vitro, and suppresses the growth of liver metastases of human colorectal cancer in vivo. Several studies of EGCG as a treatment of ectopic cancer models, particularly subcutaneous models, using various cancer cell lines, including colorectal cancer, have been reported (17-19). The major advantage of the ectopic model is that it allows the rapid screening of new cytotoxic agents. A variety of host organ environment factors have profound influences on the biological behavior of tumor cells, including the production of degradative enzymes, angio- genesis, the induction of differentiation and the transcriptional properties of genes. Studies using ectopic inoculation do not account for the organ-specific factors that influence the growth of tumor cells, and it is necessary to further examine the antitumor activity of agents in the appropriate organspecific environment (20). This is the first study on inhibiting liver metastases of human colorectal cancer by administering EGCG.

Angiogenesis is the process of generating new blood vessels, and it plays a critical role in the growth and metastasis of solid tumors by supplying nutrients and oxygen and removing waste products from the tumor (21). The inhibition of angiogenesis using chemotherapeutic agents, such as bevacizumab, is an attractive strategy for antitumor treatment (22). VEGFR2 activates various downstream signal transduction proteins, including Akt (23), which is a critical regulator of cell survival, proliferation, migration and angiogenesis (24). The interruption of VEGFR2 signaling is considered necessary for inhibition of tumor angiogenesis and tumor growth. In the present study, we demonstrated that EGCG inhibited the expression of VEGFR2 and the phosphorylation of Akt in vitro and that it suppressed angiogenesis and the growth of liver metastases in vivo.

p38 is a member of the MAPK family that is activated in response to a variety of cellular stresses including osmotic shock, inflammatory cytokines, lipopolysaccharide and UV light (25). Once phosphorylated, p38 interacts with a wide range of substrates and acts on inflammatory responses, cell differentiation, cell cycle arrest, senescence, apoptosis and 
other cell pathways (26). Anticancer drugs, such as irinotecan and oxaliplatin, have been reported to activate p38 in colon cancer cells $(27,28)$. EGCG can induce apoptosis in human colon adenocarcinoma cells by activating JNK and p38 MAPK (16). In the present study, we demonstrated that EGCG promoted the phosphorylation of $\mathrm{p} 38$ and induced apoptosis.

Cases of hepatotoxicity have been associated with the consumption of high doses of dietary supplements containing green tea (29), and a high oral dose of EGCG has been reported to induce hepatotoxicity in mice (30). In the present study, EGCG administration did not induce hepatotoxicity and weight loss. EGCG preferentially inhibits the growth of human colorectal and liver cancer cells in comparison to normal colon epithelial cells and hepatocytes $(31,32)$. Taken together, the administration of an appropriate dose of EGCG has little influence on normal cells and effectively controls liver metastases of colorectal cancer with few side-effects.

In the present study, EGCG inhibited liver metastases of human colorectal cancer in a mouse model.EGCG was recently reported to have a synergistic antitumor effect in combination with existing anticancer drugs, such as 5-fluorouracil (5-FU), capecitabine and oxaliplatin (33-35). Both 5-FU and oxaliplatin are key drugs for treating patients with liver metastases of colorectal cancer (9), and EGCG treatment in combination with these drugs has the potential to prolong survival in patients with liver metastases of colorectal cancer. Green tea, which contains abundant EGCG, is a commonly consumed beverage worldwide, and the oral intake of EGCG is reported to inhibit the growth of colorectal cancer in vivo (17). In a pilot study, supplementation with green tea extract prevented the development of metachronous colorectal adenomas (36). Collectively, EGCG may be suitable not only for chemotherapy for patients with liver metastases of colorectal cancer in combination with existing anticancer drugs, but also for oral adjuvant therapy for patients after colorectal cancer surgery.

In conclusion, EGCG inhibits cell proliferation and induces apoptosis in human colorectal cancer through Akt, p38 and VEGFR2, and it suppresses angiogenesis and liver metastases of colorectal cancer. EGCG may be useful in the treatment of liver metastases of human colorectal cancer.

\section{Acknowledgements}

The present study was supported in part by grants from the Ministry of Education, Culture, Sports, Science and Technology of Japan (MEXT) and Public Trust Surgery Research Fund. The authors thank Ako Takahashi for providing technical assistance.

\section{References}

1. Ferlay J, Shin HR, Bray F, Forman D, Mathers C and Parkin DM: Estimates of worldwide burden of cancer in 2008: GLOBOCAN 2008. Int J Cancer 127: 2893-2917, 2010.

2. Manfredi S, Lepage C, Hatem C, Coatmeur O, Faivre J and Bouvier AM: Epidemiology and management of liver metastases from colorectal cancer. Ann Surg 244: 254-259, 2006.

3. Hughes KS, Rosenstein RB, Songhorabodi S, et al: Resection of the liver for colorectal carcinoma metastases. A multi-institutional study of long-term survivors. Dis Colon Rectum 31: 1-4, 1988.
4. Scheele J, Stang R, Altendorf-Hofmann A and Paul M: Resection of colorectal liver metastases. World J Surg 19: 59-71, 1995.

5. Kato T, Yasui K, Hirai T, et al: Therapeutic results for hepatic metastasis of colorectal cancer with special reference to effectiveness of hepatectomy: analysis of prognostic factors for 763 cases recorded at 18 institutions. Dis Colon Rectum 46: S22-S31, 2003.

6. Yamada H, Katoh H, Kondo S, Okushiba S and Morikawa T: Repeat hepatectomy for recurrent hepatic metastases from colorectal cancer. Hepatogastroenterology 48: 828-830, 2001

7. Scheer MG, Sloots CE, van der Wilt GJ and Ruers TJ: Management of patients with asymptomatic colorectal cancer and synchronous irresectable metastases. Ann Oncol 19: 1829-1835, 2008.

8. Dy GK, Hobday TJ, Nelson G, et al: Long-term survivors of metastatic colorectal cancer treated with systemic chemotherapy alone: north central cancer treatment group review of 3811 patients, n0144. Clin Colorectal Cancer 8: 88-93, 2009.

9. Venook A: Critical evaluation of current treatments in metastatic colorectal cancer. Oncologist 10: 250-261, 2005.

10. Cabrera C, Artacho R and Gimenez R: Beneficial effects of green tea: a review. J Am Coll Nutr 25: 79-99, 2006.

11. Ji BT, Chow WH, Hsing AW, et al: Green tea consumption and the risk of pancreatic and colorectal cancers. Int J Cancer 70: 255-258, 1997.

12. Yang G, Shu XO, Li H, et al: Prospective cohort study of green tea consumption and colorectal cancer risk in women. Cancer Epidemiol Biomarkers Prev 16: 1219-1223, 2007.

13. Cabrera C, Gimenez R and Lopez MC: Determination of tea components with antioxidant activity. J Agric Food Chem 51: 4427-4435, 2003.

14. Du GJ,Zhang Z, Wen XD, et al: Epigallocatechin gallate (EGCG) is the most effective cancer chemopreventive polyphenol in green tea. Nutrients 4: 1679-1691, 2012.

15. Shimizu M, Adachi S, Masuda M, Kozawa $\mathrm{O}$ and Moriwaki $\mathrm{H}$ : Cancer chemoprevention with green tea catechins by targeting receptor tyrosine kinases. Mol Nutr Food Res 55: 832-843, 2011.

16. Chen C, Shen G, Hebbar V, Hu R, Owuor ED and Kong AN: Epigallocatechin-3-gallate-induced stress signals in HT-29 human colon adenocarcinoma cells. Carcinogenesis 24: 1369-1378, 2003.

17. Shimizu M, Shirakami Y, Sakai H, et al: (-)-Epigallocatechin gallate inhibits growth and activation of the VEGF/VEGFR axis in human colorectal cancer cells. Chem Biol Interact 185: 247-252, 2010

18. Tran PL, Kim SA, Choi HS, Yoon JH and Ahn SG: Epigallocatechin-3-gallate suppresses the expression of HSP70 and HSP90 and exhibits anti-tumor activity in vitro and in vivo. BMC Cancer 10: 276, 2010.

19. Jin H, Gong W, Zhang C and Wang S: Epigallocatechin gallate inhibits the proliferation of colorectal cancer cells by regulating Notch signaling. Onco Targets Ther 6: 145-153, 2013.

20. Hoffman RM: Orthotopic metastatic mouse models for anticancer drug discovery and evaluation: a bridge to the clinic. Invest New Drugs 17: 343-359, 1999.

21. Nishida N, Yano H, Nishida T, Kamura $T$ and Kojiro M: Angiogenesis in cancer. Vasc Health Risk Manag 2: 213-219, 2006.

22. Shih $\mathrm{T}$ and Lindley $\mathrm{C}$ : Bevacizumab: an angiogenesis inhibitor for the treatment of solid malignancies. Clin Ther 28: 1779-1802, 2006.

23. Jiang BH and Liu LZ: AKT signaling in regulating angiogenesis. Curr Cancer Drug Targets 8: 19-26, 2008.

24. Manning BD and Cantley LC: AKT/PKB signaling: navigating downstream. Cell 129: 1261-1274, 2007.

25. Zarubin T and Han J: Activation and signaling of the p38 MAP kinase pathway. Cell Res 15: 11-18, 2005.

26. Shi Y and Gaestel M: In the cellular garden of forking paths: how p38 MAPKs signal for downstream assistance. Biol Chem 383: 1519-1536, 2002.

27. Rudolf E, Kralova V, Rudolf K and John S: The role of p38 in irinotecan-induced DNA damage and apoptosis of colon cancer cells. Mutat Res 741-742: 27-34, 2013.

28. Liu HF, Hu HC and Chao JI: Oxaliplatin down-regulates survivin by $\mathrm{p} 38$ MAP kinase and proteasome in human colon cancer cells. Chem Biol Interact 188: 535-545, 2010.

29. Mazzanti G, Menniti-Ippolito F, Moro PA, et al: Hepatotoxicity from green tea: a review of the literature and two unpublished cases. Eur J Clin Pharmacol 65: 331-341, 2009. 
30. Lambert JD, Kennett MJ, Sang S, Reuhl KR, Ju J and Yang CS: Hepatotoxicity of high oral dose (-)-epigallocatechin-3-gallate in mice. Food Chem Toxicol 48: 409-416, 2010.

31. Shimizu M, Deguchi A, Lim JT, Moriwaki H, Kopelovich L and Weinstein IB: (-)-Epigallocatechin gallate and polyphenon E inhibit growth and activation of the epidermal growth factor receptor and human epidermal growth factor receptor-2 signaling pathways in human colon cancer cells. Clin Cancer Res 11: 2735-2746, 2005.

32. Shimizu M, Shirakami Y, Sakai H, et al: EGCG inhibits activation of the insulin-like growth factor (IGF)/IGF-1 receptor axis in human hepatocellular carcinoma cells. Cancer Lett 262: 10-18, 2008.

33. Yunos NM, Beale P, Yu JQ and Huq F: Synergism from the combination of oxaliplatin with selected phytochemicals in human ovarian cancer cell lines. Anticancer Res 31: 4283-4289, 2011.
34. Yang XW, Wang XL, Cao LQ, et al: Green tea polyphenol epigallocatechin-3-gallate enhances 5-fluorouracil-induced cell growth inhibition of hepatocellular carcinoma cells. Hepatol Res 42: 494-501, 2012.

35. Wu H, Xin Y, Xu C and Xiao Y: Capecitabine combined with (-)-epigallocatechin-3-gallate inhibits angiogenesis and tumor growth in nude mice with gastric cancer xenografts. Exp Ther Med 3: 650-654, 2012

36. Shimizu M, Fukutomi Y, Ninomiya M, et al: Green tea extracts for the prevention of metachronous colorectal adenomas: a pilot study. Cancer Epidemiol Biomarkers Prev 17: 3020-3025, 2008. 